\title{
El apixaban se asocia a un menor riesgo de sangrado que los antagonistas de la vitamina $\mathrm{K}$
}

Apixaban is associated with a lower risk of bleeding than vitamin $\mathrm{K}$ antagonists

Touma L, y col. The American Journal of Cardiology 2015;115:533-541.

\section{Objetivos}

Comparar el riesgo de sangrado y de mortalidad por todas las causas entre apixaban y los antagonistas de la vitamina $K(A V K)$, drogas utilizadas para reducir el riesgo de accidente cerebrovascular y tromboembolismo en pacientes con fibrilación auricular y en aquellos cursando el post-operatorio de cirugía de reemplazo de rodilla o cadera.

Fuentes de datos, selección de estudios y extracción de datos Se realizaron búsquedas en MEDLINE, EMBASE, Cochrane Library. Se incluyeron ensayos controlados aleatorizados (ECA) publicados, en inglés o francés, desde 2014, que hayan utilizado apixaban a dosis recomendadas $(2,5 \circ 5 \mathrm{mg}$, dos veces al día) o AVK en pacientes mayores de 18 años. Se excluyeron pacientes con enfermedades malignas.

No se describe el proceso de selección, pero dos revisores extrajeron de forma independiente eventos de sangrado, mayor o menor pero clínicamente relevante y cualquier causa de muerte.

\section{Resultados Principales}

Se aleatorizaron 25.576 pacientes en los 5 ensayos incluidos: ARISTOTLE (apixaban versus vs. warfarina en el tratamiento de fibrilación auricular no valvular), AMPLIFY (apixaban vs. warfarina para el tratamiento inicial de embolia pulmonar y trombosis venosa profunda con heparina de bajo peso molecular (HBPM) como tratamiento inicial, ARISTOTLE-J (apixaban vs. warfarina en fibrilación auricular en pacientes japoneses), APROPOS (apixaban vs. warfarina para profilaxis de tromboembolismo en cirugía de reemplazo total de rodilla), BOTTICELLI (apixaban vs warfarina para trombosis venosa profunda sintomática precedido por HBPM). La duración mediana del tratamiento fue variable entre 10 y 657 días, y el seguimiento, entre 40 días y 657 días. Todos los ensayos tuvieron bajo riesgo de sesgo.

Los resultados principales se resumen en la Tabla 1.

Tabla 1. Resultados principales de la comparación de apixaban vs antagonistas de vitamina $\mathrm{K}$

\begin{tabular}{l|c|c|c|c|c}
\multicolumn{1}{c|}{ Resultado } & Apixaban & AVK & RRR (IC95\%) & NNT (IC95\%) & $\mathbf{R}^{\mathbf{2}}$ \\
\hline Cualquier sangrado & $23 \%$ & $32 \%$ & $27 \%(10 \mathrm{a} 41)$ & $12(8 \mathrm{a} 32)$ & $84 \%$ \\
\hline Sangrado mayor o clínicamente relevante & $5,8 \%$ & $9,6 \%$ & $40 \%(12 \mathrm{a} 60)$ & $26(18 \mathrm{a} 87)$ & $82 \%$ \\
\hline Sangrado intracraneal & $0,5 \%$ & $1,1 \%$ & $58 \%(42 \mathrm{a} 69)$ & $158(133 \mathrm{a} 219)$ & $0 \%$ \\
\hline Mortalidad por todas las causas & $5,3 \%$ & $6,0 \%$ & $11 \%(1 \mathrm{a} 19)$ & $153(89 \mathrm{a} 1676)$ & $0 \%$ \\
\hline
\end{tabular}

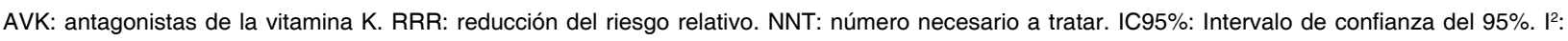
heterogeneidad.

\section{Conclusiones}

El Apixaban está asociado con menor riesgo de sangrado y menor mortalidad por todas las causas comparado con los AVK.
Fuente de financiamiento/conflicto de interés de los autores: No reportado. Los autores no tienen conflictos de interés que reportar.

\section{Comentario}

El Apixaban (inhibidor directo del Factor Xa) es uno de los nuevos anticoagulantes que se utiliza como una alternativa de los AVK. Los anticoagulantes directos tienen varias ventajas farmacológicas sobre los $\mathrm{AVK}$, incluyendo rápido inicio de acción, vida media más corta (de 7 a 14 hs en personas sanas). Se administran a dosis fijas sin necesidad de monitoreo de laboratorio, a diferencia de la warfarina que requiere monitoreo de la razón internacional normatizada (RIN) y ajustes periódicos de la dosis. En ECA con buen manejo de la anticoagulación (RIN en rango de 2 a 3 en más de $60 \%$ del tiempo), los anticoagulantes directos fueron no inferiores, y en algunos casos superiores, a warfarina dosis-ajustada para la prevención y tratamiento de la trombosis ${ }^{1}$. Comparados con warfarina, los anticoagulantes directos redu- jeron $28 \%$ del riesgo de sangrado mayor y $50 \%$ las tasas de sangrado intracraneal ${ }^{2}$. A pesar de todo, los médicos siguen prefiriendo los AVK debido a la "falta de antídoto" para los nuevos anticoagulantes y a que los agentes no específicos para revertir la anticoagulación, como el protromplex, el plasma fresco o el factor VIIa, no reducirían el efecto anticoagulante de estos medicamentos in vitro, en modelos animales o en voluntarios sanos ${ }^{3}$.

\section{Conclusiones del comentador}

A medida que aumente la experiencia clínica en el uso de los nuevos anticoagulantes, estos serán cada vez más indicados, ya que a todos los beneficios mencionados anteriormente se suma el menor riesgo de sangrado demostrado por la evidencia.

María Paula Cárdenas [ Hospital Italiano de Buenos Aires, maria.cardenas@ hospitalitaliano.org.ar ]

Cárdenas MP. El apixaban se asocia a un menor riesgo de sangrado que los antagonistas de la vitamina K. Evid Act Pract Ambul 2018;21(2):54. Comentado de Lahoud T, y col. A Meta-Analysis of Randomized Controlled Trials of the Risk of Bleeding With Apixaban Versus Vitamin K Antagonists. The American Journal of Cardiology 2014;115(4)533-541. PMID: 25547937

\section{Referencias}

1. Bauer KA.Targeted Anti-Anticoagulants. N Engl J Med. 2015 Aug 6;373(6):569-71

2.Chai-Adisaksopha C, y col. The impact of bleeding complications in patients receiving target-specific oral anticoagulants: a systematic review and meta-analysis. Blood 2014;124:2450-8.

3. Bauer KA. Reversal of antithrombotic agents. Am J Hematol 2012;87:Suppl 1:S119-S126 